\title{
Femtosecond-laser-based synthesis of ultrastable microwave signals from optical frequency references
}

\author{
A. Bartels, S. A. Diddams, C. W. Oates, G. Wilpers, J. C. Bergquist, W. H. Oskay, and L. Hollberg \\ Time and Frequency Division, National Institute of Standards and Technology, 325 Broadway, M.S. 847, Boulder, \\ Colorado 80305
}

\begin{abstract}
We use femtosecond laser frequency combs to convert optical frequency references to the microwave domain, where we demonstrate the synthesis of $10-\mathrm{GHz}$ signals having a fractional frequency instability of $\leqslant 3.5$ $\times 10^{-15}$ at a 1 -s averaging time, limited by the optical reference. The residual instability and phase noise of the femtosecond-laser-based frequency synthesizers are $6.5 \times 10^{-16}$ at $1 \mathrm{~s}$ and $-98 \mathrm{dBc} / \mathrm{Hz}$ at a $1-\mathrm{Hz}$ offset from the $10-\mathrm{GHz}$ carrier, respectively. The timing jitter of the microwave signals is $3.3 \mathrm{fs}$.
\end{abstract}

Optical frequency standards based on neutral atoms, ions, or molecules are readily capable of achieving lower instabilities than the best current microwave standards; in the future they may achieve fractional frequency instabilities as low as $1 \times 10^{-16} \tau^{-1 / 2}(\tau$ is the averaging time in seconds) with uncertainties approaching $10^{-18} \cdot{ }^{1-3}$ The local oscillators of optical frequency standards are $\mathrm{cw}$ lasers that are stabilized to high-finesse optical cavities. An instability of 4 $\times 10^{-16}$ for averaging times from 1 to $30 \mathrm{~s}$ has been demonstrated. ${ }^{4}$ To create an optical atomic clock with a microwave output signal possessing the stability and uncertainty of an optical frequency standard, the frequency of the local oscillator must be phasecoherently divided by a factor of $\approx 10^{6}$. Femtosecond laser frequency combs (FLFCs) are a convenient means of performing this task, as has been demonstrated previously. ${ }^{5}$ In that experiment the measured fractional frequency instability of the microwave output signal at $1 \mathrm{GHz}$ was limited to $2.5 \times 10^{-13}$ at $1 \mathrm{~s}$ by the hydrogen maser used for comparison. More recently, upper limits on the residual instability of FLFCs were established by comparison of two such systems that share a common optical reference. Specifically, optical pulse trains at $1 \mathrm{GHz}$ with a residual instability of $2.3 \times 10^{-15}$ at $1 \mathrm{~s}$, limited by measurement resolution, have been demonstrated. ${ }^{6}$ Microwave signals obtained by photodetection of those pulse trains had an instability of $2 \times 10^{-14}$ at a 1 -s averaging time, limited by excess noise from photodetection and electronic processing. Major contributions to this excess noise are identified and eliminated in the present work. In this Letter we use two FLFCs to synthesize $10-\mathrm{GHz}$ microwave signals with an instability of $\leqslant 3.5 \times 10^{-15}$ at $1 \mathrm{~s}$, referenced to two independent optical frequency references. We determine an upper limit to the residual fractional frequency instability and single-sideband phase noise of the FLFCs by comparing two systems that share a common optical reference.
Two cw lasers at 657 and $563 \mathrm{~nm}$ that are stabilized to their respective high-finesse optical cavities are used as optical references. These lasers usually serve as the local oscillators of the National Institute of Standards and Technology (NIST) $\mathrm{Ca}$ and $\mathrm{Hg}^{+}$-ion optical frequency standards. When not locked to their atomic references (as is the case here), they have drift rates of $\leqslant 10$ and $\leqslant 1 \mathrm{~Hz} / \mathrm{s}$, respectively. ${ }^{2,4}$ The core of each of our two nearly identical FLFC systems (referred to by indices $i=1,2$ ) is a femtosecond laser with a repetition rate of $1 \mathrm{GHz}$ that emits a broadband continuum. ${ }^{7}$ For each system we phase lock a heterodyne beat signal at frequency $f_{b, i}$ between the optical reference (with frequency $f_{\mathrm{LO}, i}$ ) and the neighboring component of the FLFC (with mode number $n_{i}$ ) to a stable RF source. The carrier-envelope offset frequency $f_{0, i}$ of each FLFC is phase locked to a second $\mathrm{RF}$ source such that the repetition rates are phase-coherently linked to the optical reference and given by $f_{R, i}=\left(f_{\mathrm{LO}, i}-f_{b, i}-f_{0, i}\right) / n_{i}$. trains from the FLFCs are detected with individual fiber-coupled InGaAs p-i-n photodiodes; no active stabilization measures for the direct photocurrent were implemented. Narrow-bandpass filters select the tenth harmonics of the repetition rates. The signal strength at $10 \mathrm{GHz}$ obtained directly from the photodetectors is $-20 \mathrm{dBm}$. These signals were amplified and supplied to a double-balanced mixer whose output provides $\Delta f_{R_{10}}=10 \times\left(f_{R, 1}-f_{R, 2}\right)$, which is used to characterize the instability and phase noise of the $10-\mathrm{GHz}$ signals.

We begin by creating two truly independent $10-\mathrm{GHz}$ signals by linking one FLFC to the $657-\mathrm{nm}$ reference and the other to the $563-\mathrm{nm}$ reference $\left(f_{\mathrm{LO}, 1}=456 \mathrm{THz}\right.$ and $\left.f_{\mathrm{LO}, 2}=532 \mathrm{THz}\right)$. We set $\Delta f_{R_{10}}$ to be a few kilohertz by our choice of $n_{i}, f_{0, i}$, and $f_{b, i}$ and acquired a series of frequency readings with a highresolution counter at different gate times $\tau$. For each data series we computed the Allan deviation 
$\sigma_{y, 10 \mathrm{GHz}}(\tau)$, a measure of fractional frequency instability of the $10-\mathrm{GHz}$ signals. ${ }^{9}$ A linear drift was removed from the data to account for the drift of the optical reference cavities. Curve a in Fig. 1 shows the obtained $\sigma_{y, 10 \mathrm{GHz}}(\tau)$. At the same time the instability of the 657-nm local oscillator was measured as shown in curve b (see Ref. 5 for experimental details). The instability of the $10-\mathrm{GHz}$ signals clearly tracks that of the $657-\mathrm{nm}$ source with a value of $3.5 \times 10^{-15}$ at $1 \mathrm{~s}$; the instability of the $563-\mathrm{nm}$ local oscillator is known to be smaller than that of the $657-\mathrm{nm}$ laser. ${ }^{4}$ The nonlinear drift of the $657-\mathrm{nm}$ source gives rise to the instability increase at longer gate times. For the case that the local oscillators are locked to their atomic frequency reference, we anticipate that the instability of the $10-\mathrm{GHz}$ signals will decrease as $1 / \sqrt{\tau}$ at longer gate times, limited only by the residual instability of the FLFCs (see below).

To measure the excess (or residual) noise of the optical-to-microwave division with the FLFCs, we repeated the above measurement with a common reference scheme in which both FLFCs are locked to the $657-\mathrm{nm}$ local oscillator (i.e., $f_{\mathrm{LO}, 1}=f_{\mathrm{LO}, 2} \approx 456 \mathrm{THz}$ ). In this case any instability of the optical reference is common to both $10-\mathrm{GHz}$ signals and does not significantly contribute to $\sigma_{y, 10 \mathrm{GHz}}(\tau)$. Curve d in Fig. 1 shows the obtained $\sigma_{y, 10 \mathrm{GHz}}(\tau)$. We applied a factor of $1 / \sqrt{2}$ to these data, assuming that both synthesizers contribute equally. The data initially exhibited a $\tau^{-1}$ behavior. However, at averaging times greater than $1 \mathrm{~s}$ the $\sigma_{y, 10 \mathrm{GHz}}(\tau)$ improve slower than $\tau^{-1}$, suggesting that a slow phase variation with a period of $\approx 10 \mathrm{~s}$ is present. The instability at $1 \mathrm{~s}$ is $6.5 \times 10^{-16}$, representing a 30 -fold improvement over earlier results. ${ }^{6}$ Most of this improvement comes from eliminating several slow environmental perturbations that caused variations of the optical power incident on the photodetectors and led to excess phase noise through amplitude-to-phase (AM-PM) coupling. ${ }^{10}$ Nonetheless, we believe that AM-PM coupling in the photo-

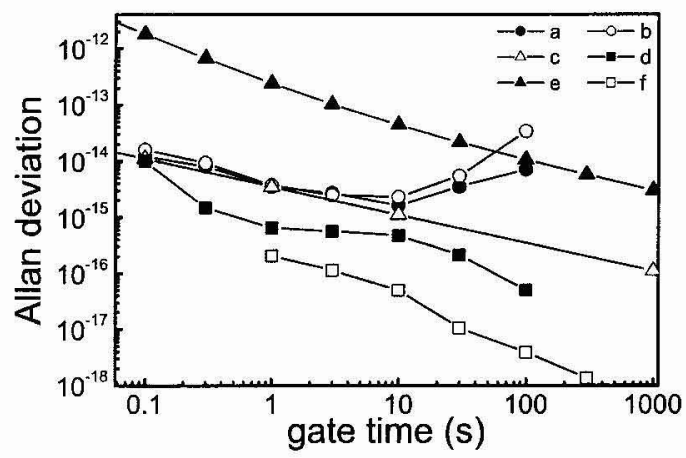

Fig. 1. Allan deviation measurements taken at microwave (filled symbols) and optical (open symbols) carrier frequencies: Curve a, $\sigma_{y, 10 \mathrm{GHz}}(\tau)$ when the $10-\mathrm{GHz}$ signals are synthesized from two independent cavity-stabilized cw lasers; curve b, instability of the 657-nm cw laser; curve c, current demonstrated upper limit for the NIST $\mathrm{Ca}$ and $\mathrm{Hg}^{+}$-ion optical frequency standards ${ }^{5}$; curve $\mathrm{d}$, residual $\sigma_{y, 10 \mathrm{GHz}}(\tau)$ when a common optical reference is used; curve e, state-of-the-art hydrogen maser; curve f, FLFCs in the optical domain.
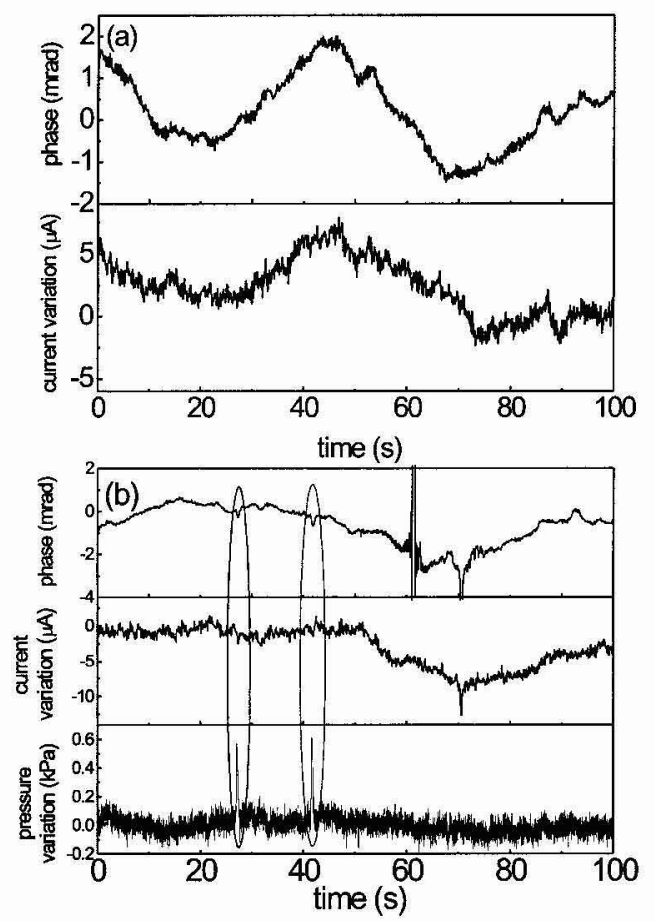

Fig. 2. (a) Phase between two $10-\mathrm{GHz}$ signals derived from a common optical reference at equal $f_{R, i}$ (upper trace) and variation of the photocurrent through one of the photodetectors from its mean value (lower trace). (b) Upper and middle trace show data as in (a), and the lower trace depicts variations of ambient pressure from the mean value. Ellipses mark times when a laboratory door was opened. At 61 and $70 \mathrm{~s}$ the system was deliberately perturbed.

detectors is still a principal limitation. The upper trace in Fig. 2(a) shows the DC output of the mixer around its zero crossing scaled to represent the phase between the input signals when the $f_{R, i}$ are set equal. The lower trace reveals prominent variations of the detected average photocurrent through one of the photodetectors. The strong correlation between the two curves suggests that the arrival time at the counter of the current pulse generated by the optical pulse varies linearly with the incident power. ${ }^{10} \mathrm{~A}$ current-to-timing coupling coefficient of $\approx 6 \mathrm{ps} / \mathrm{mA}$ can be extracted (at the operating point of $1.7 \mathrm{~mA}$ of direct current). The slow phase dynamics occur with periods of a few tens of seconds with a slope of $\approx 100 \mu \mathrm{rad} / \mathrm{s}$, which is in agreement with the plateau at $\approx 10^{-15}$ between 1 and $30 \mathrm{~s}$ in curve $d$ of Fig. 1 . Other sources of AM-PM coupling, e.g., the amplifiers or the mixer, might also contribute to the data.

The origin of the photocurrent modulation present on one photodetector with an amplitude of $0.3 \%$ and a period of $\approx 50 \mathrm{~s}$ is presently unknown. We have ruled out temperature variations of the laser housing, the crystal, and the ambient environment. We tested the sensitivity of the FLFCs to ambient pressure as shown in Fig. 2(b) but detected no correlation between pressure (lower trace) and the slow dynamics on phase and photocurrent (upper and middle traces). However, abrupt pressure changes can affect the amount of light coupled to the photodetectors, 
causing phase variations of the $10-\mathrm{GHz}$ signals. This becomes evident in Fig. 2(b), in which at approximately 27 and $42 \mathrm{~s}$ a laboratory door was rapidly opened. Additionally, at $61 \mathrm{~s}$ the microwave cable leading to the mixer and at $70 \mathrm{~s}$ the fiber guiding light to the photodetector were slightly touched, creating phase excursions of tens of milliradians.

Despite the remaining slow phase variations, the microwave signals generated by the optically referenced FLFCs have an instability comparable with or better than that of the most-stable microwave signals obtained from any source. They are approximately 2 orders of magnitude more stable than the output of a state-of-the-art hydrogen maser for averaging times up to $10 \mathrm{~s}$ (see curves a and e in Fig. 1). From curve d in Fig. 1 we infer that we are readily able to transfer the current stability of NIST's $\mathrm{Ca}$ and $\mathrm{Hg}^{+}$-ion optical frequency standards (curve c) to the microwave domain. Curve $f$ in Fig. 1 shows the instability of the FLFCs measured in the optical domain $\left(2 \times 10^{-16}\right.$ at $1 \mathrm{~s}$; see Ref. 11 for details), representing what the systems will be capable of in the microwave domain when the remaining excess noise is suppressed.

To measure the single-sideband phase noise $L(f)$ of the $10-\mathrm{GHz}$ signals, we used the common reference scheme with equal $f_{R, i}$ and adjusted the phase between the signals such that the DC signal from the mixer was at its zero crossing for minimum amplitude sensitivity and maximum phase sensitivity. This signal was then analyzed with a fast Fourier transform analyzer to yield $L(f)$ as shown in Fig. 3. At a $1-\mathrm{Hz}$ offset from the $10-\mathrm{GHz}$ carrier the phase noise is $-98 \mathrm{dBc} / \mathrm{Hz}$. The timing jitter of the $10-\mathrm{GHz}$ microwave signals integrated between $0.1 \mathrm{~Hz}$ and $1 \mathrm{MHz}$ is as low as $3.3 \mathrm{fs}$, relative to each other and the optical reference. The dashed curve shows the phase noise of the amplifiers used after the photodetectors and before the mixer. At offset frequencies above $100 \mathrm{~Hz}$, amplifier noise is a significant limiting factor to our measurement, which thus yields only an upper limit at higher frequencies. To our knowledge, the phase noise of our FLFC-based synthesizers close to the carrier $(<100 \mathrm{~Hz})$ is lower than what has been

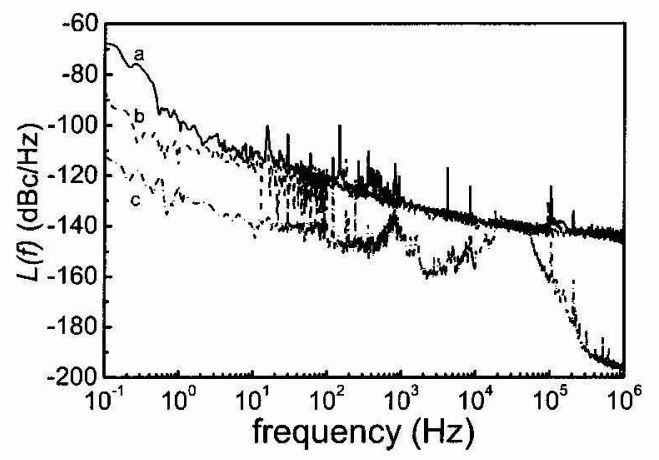

Fig. 3. Curve a, Residual phase noise of $10-\mathrm{GHz}$ microwave signals derived from two FLFCs locked to a common reference; curve b, phase noise floor of the microwave amplifiers; curve $c$, residual phase noise of the FLFCs in the optical domain. demonstrated for any source of $10-\mathrm{GHz}$ signals. For example, a sapphire cavity oscillator might have $L(10 \mathrm{MHz})=-178 \mathrm{dBc} / \mathrm{Hz}$, but at a low Fourier frequency it is typically steered to a quartz oscillator such that $L(1 \mathrm{~Hz}) \approx-65 \mathrm{dBc} / \mathrm{Hz} .{ }^{12}$ The dasheddotted curve shows the phase noise of the FLFC components in the optical domain scaled to a $10-\mathrm{GHz}$ carrier wave, representing what the FLFCs could achieve at $10 \mathrm{GHz}$ if slow phase variations and amplifier noise were reduced. ${ }^{11}$

In conclusion, we have used optically referenced FLFC-based frequency synthesizers to create $10-\mathrm{GHz}$ microwave signals with an instability of 3.5 $\times 10^{-15}$ at $1 \mathrm{~s}$. The residual instability of the FLFCbased optical-to-microwave division process is 6.5 $\times 10^{-16}$ at $1 \mathrm{~s}$, limited by AM-PM coupling. The residual single-sideband phase noise at a $1-\mathrm{Hz}$ offset from the $10-\mathrm{GHz}$ carrier is $-98 \mathrm{dBc} / \mathrm{Hz}$, currently limited by amplifier noise at offset frequencies $>100 \mathrm{~Hz}$. Our results reflect the stability that can be expected for the microwave output of future optical atomic clocks and provide an upper limit to the phase noise of microwave signals that can be synthesized from optical frequency references by means of a FLFC.

We are grateful to R. Fox, J. McFerran, and E. Ivanov for their contributions. This work was funded by NASA, NIST, and the Defense Advanced Research Projects Agency. A. Bartels's e-mail address is bartels@gigaoptics.com; S. A. Diddams's is sdiddams@boulder.nist.gov.

\section{References}

1. R. J. Rafac, B. C. Young, J. A. Beall, W. M. Itano, D. J. Wineland, and J. C. Bergquist, Phys. Rev. Lett. 85, $2462(2000)$.

2. C. W. Oates, E. A. Curtis, and L. Hollberg, Opt. Lett. 25, 1603 (2000).

3. J. Ye, L.-S. Ma, and J. L. Hall, Phys. Rev. Lett. 87, 270801 (2001).

4. B. C. Young, F. C. Cruz, W. M. Itano, and J. C. Bergquist, Phys. Rev. Lett. 82, 3799 (1999).

5. S. A. Diddams, Th. Udem, J. C. Bergquist, E. A. Curtis, R. E. Drullinger, L. Hollberg, W. M. Itano, W. D. Lee, C. W. Oates, K. R. Vogel, and D. J. Wineland, Science 293, 825 (2001).

6. A. Bartels, S. A. Diddams, T. M. Ramond, and L. Hollberg, Opt. Lett. 28, 663 (2003).

7. A. Bartels and H. Kurz, Opt. Lett. 27, 1839 (2002).

8. T. M. Ramond, S. A. Diddams, L. Hollberg, and A. Bartels, Opt. Lett. 27, 1842 (2002).

9. D. W. Allan, IEEE Trans. Ultrason. Ferroelectr. Freq. Control 34, 647 (1987).

10. E. N. Ivanov, S. A. Diddams, and L. Hollberg, IEEE J. Sel. Top. Quantum Electron. 9, 1059 (2003).

11. A. Bartels, C. W. Oates, L. Hollberg, and S. A. Diddams, Opt. Lett. 29, 1081 (2004).

12. Advertised data for model SLCO of Poseidon Scientific Instruments, Australia, and personal communication with E. N. Ivanov, University of Western Australia. Trade name is mentioned for scientific clarity. NIST does not endorse this product; others might be equally or better suited. 